\title{
El declive del estado de resultados tradicional ante la irrupción actual del excedente limpio Perspectiva histórica y conceptual
}

\section{Francisco Sousa}

\section{Fernández.}

Profesor-investigador, Departamento de Administración de Empresas, Universidad de Cantabria.sousaf@unican.es

\section{Resumen}

Con la adopción del resultado global (comprehensive income) a partir de la década de los noventa del siglo XX por los organismos emisores de normas contables más relevantes en el concierto mundial, entre los que cabe destacar el International Accounting Standards Board (IASB) y el Financial Accounting Standards Board (FASB), estamos asistiendo a un posicionamiento a favor del enfoque del excedente limpio. Como consecuencia el resultado empresarial queda más vinculado a las oscilaciones de los precios de los títulos y a los tipos de cambio, frente al enfoque del excedente sucio que nos lleva a la determinación del mismo desde una óptica más restrictiva y, por ello, mucho menos conectado con la realidad de los mercados.

En este trabajo nos planteamos como primer objetivo el estudio desde una óptica histórica y conceptual del antiguo y controvertido debate del enfoque del excedente limpio versus el enfoque del excedente sucio, circunscrito esencialmente a los países del área de influencia anglosajona. Posteriormente, queremos constatar que con la revisión de la Norma Internacional de Contabilidad 1 (NIC 1) del International Accounting Standards Board (IASB) en el año 2007 cobra más fuerza el resultado global; con ello se confirman los planteamientos a favor del enfoque del excedente limpio, lo que origina la desaparición 
del estado de resultados tradicional del conjunto completo de estados financieros si las empresas, según dicha norma, optan por revelarlo en el estado de resultados globales; todo ello con el propósito de reportar más utilidad a los inversionistas, quienes son considerados usuarios primordiales en los modelos contables anglosajones a los que tendemos.

Palabras clave: excedente limpio versus excedente sucio, óptica histórica y conceptual, declive del estado de resultados tradicional, estado de resultados globales, Norma Internacional de Contabilidad 1 (NIC 1) 2007.

\title{
The decline of the traditional statement of income in the face of the recent rise of clean surplus accounting: a historical and conceptual perspective
}

\begin{abstract}
With the adoption of comprehensive income in the 1990's by the most important accounting standards boards, most notably the IASB and the FASB, we are witnessing a shift towards a clean surplus accounting. The comprehensive view of income measures income in terms or both the changes in the market value of a firm's assets and liabilities as well as the profit/ loss resulting from a firm's transactions. By contrast, so-called "dirty surplus" accounting measures income only on the basis of a firm's transactions; thus, it is much less connected with market reality.
\end{abstract}

The primary objective of this paper is to provide a historical and conceptual examination of the "clean surplus versus dirty surplus" controversy as it has transpired within AngloSaxon influenced countries. Additionally, we verify that comprehensive income has become more relevant with the revision of IASB's IAS 1 in 2007. This statement allows firms to incorporate the traditional profit and loss account into a Statement of Comprehensive Income. The objective of this change is to provide investors, the primary users of reference in Anglo-Saxon accounting models, with more useful and relevant information. This objective is important since we are trending toward the Anglo-Saxon model.

Keywords: clean surplus versus dirty surplus, historical and conceptual approach, decline of profit and loss account, statement of comprehensive income, IAS 12007.

\section{Introducción}

Desde el siglo XIX, y particularmente a lo largo del siglo XX, hemos asistido a un controvertido debate, aún abierto en la actualidad, con respecto a qué partidas 
han de configurar el resultado empresarial y, por tanto, deben incluirse en el estado de resultados con la finalidad de satisfacer adecuadamente las necesidades de los usuarios de la información financiera.

Por una parte, tenemos a los defensores de un estado de resultados globales que afirman que en éste se incluyan no solamente los ingresos y los gastos reconocidos tradicionalmente, sino también las pérdidas y ganancias derivadas de las variaciones en el valor razonable de los elementos del balance. Para ello argumentan que un estado de resultados en el que se incluyan los anteriores elementos originará una mayor relevancia para los interesados en la marcha de las entidades económicas, en particular para los inversionistas, considerados en el marco conceptual como usuarios de referencia. Esta línea de pensamiento en terminología anglosajona se corresponde con el enfoque denominado clean surplus.

Por otro lado, existen planteamientos más conservadores conocidos en términos anglosajones como enfoque dirty surplus, en el que según sus partidarios el resultado contable a revelar en el estado de resultados tradicional, ha de configurarse sobre la base de los principios tradicionales del precio de adquisición o costo histórico y prudencia, con un claro posicionamiento a favor de la fiabilidad. De acuerdo con este enfoque los cambios en el valor razonable de los elementos del balance, en caso de contemplarse, se trasladarían al patrimonio neto o se informaría únicamente de ellos en las notas y, en consecuencia, no se reconocerían en el estado de resultados hasta su realización.

La traducción literal a la lengua española de los términos clean surplus y dirty surplus, sería "excedente limpio" y "excedente sucio", respectivamente, los cuales son suficientemente gráficos para expresar la diferencia entre esas dos líneas de pensamiento contrapuestas. El primer término en su concepción más genuina hace referencia al concepto de resultado global (comprehensive income) de la empresa por todos los conceptos, que se configura teniendo en cuenta las variaciones en la valoración autónoma de los activos y pasivos en un determinado ejercicio, excluyendo las operaciones con los propietarios. El segundo término responde a un concepto de excedente económico empresarial mucho más limitado que supone una "contaminación" del resultado al omitirse de esa forma información relevante en el estado de resultados en aplicación de los principios antes aludidos. 
Previa acotación de los fundamentos teóricos esenciales sobre los que se sustentan el resultado global y el resultado realizado tradicional, o un enfoque equivalente, que proveen el excedente limpio y el excedente sucio, respectivamente, planteamos como objetivo estudiar desde una perspectiva histórica y conceptual el controvertido debate con respecto a ambos enfoques, para lo que nos apoyamos esencialmente en la literatura contable anglosajona. Posteriormente, queremos constatar el estado de la cuestión en la actualidad, centrándonos en las novedades que incorpora al respecto la Norma Internacional de Contabilidad 1 (NIC 1) del International Accounting Standards Board (IASB) revisada en el año 2007.

Podemos anticipar que el referido debate tiene sus orígenes en el siglo XIX, aunque a lo largo del siglo XX se encuentran una serie de periodos interrumpidos en el sentido de que estamos ante la preeminencia del enfoque del excedente limpio en unas etapas concretas y la supremacía del enfoque del excedente sucio en otras.

Cabe destacar que desde la década de los noventa del pasado siglo con la aprobación de la normativa del resultado global por los principales organismos reguladores, entre los que se encuentran el Financial Reporting Standard 3 (FRS 3) (1992, revisado en 1993, 1999 y 2007) del Accounting Standards Board (ASB), el Statement of Financial Accounting Standards 130 (SFAS 130) (1997) del Financial Accounting Standards Board (FASB) y la Norma Internacional de Contabilidad 1 (NIC 1) (1999 revisada en 2003) del International Accounting Standards Board (IASB) y, principalmente, con la revisión de dicha norma internacional en 2007 se confirman los planteamientos a favor del enfoque del excedente limpio para así reportar una mayor utilidad a los inversionistas considerados en el marco conceptual como usuarios de referencia.

Lo anterior supone el hecho tan relevante para la contabilidad del ocaso del estado de resultados tradicional al quedar subsumido en el estado de resultados globales, que lo integra. 


\section{Soporte conceptual del resultado global frente al resultado realizado tradi- cional}

El FASB fue el organismo pionero en incorporar el concepto de resultado global en el SFAC 3 (1980), reemplazado por el SFAC 6 (1985), que en su párrafo 70 se define como: ${ }^{1}$

Los cambios en el patrimonio neto de una empresa de negocios durante un periodo, que tienen su origen en transacciones y otros sucesos y circunstancias no relacionados con operaciones con la propiedad. Incluye todos los cambios en el neto durante un periodo, excepto aquellos resultantes de las inversiones de los propietarios y de las distribuciones de fondos a los mismos.

Esta conceptuación nos lleva a posicionarnos en la teoría del excedente limpio (Feltham y Ohlson, 1995; Brief y Peasnell, 1996; Beale y Davey, 2000; Mattessich, 2002, entre otros), conforme a la cual el resultado contable se determina comparando el valor en libros del patrimonio neto al final de un ejercicio económico con el registrado al inicio del mismo, una vez eliminadas las operaciones con los propietarios.

De este modo, tal como señalan García-Ayuso y Monterrey: "[...] el mantenimiento en el futuro de la condición de excedente limpio asegura que todos los acontecimientos relevantes desde el punto de vista del valor serán absorbidos por los resultados contables" (1998: 755).

Sin embargo, Mattessich afirma que para este cálculo sea consistente no se debe estar ante reclamaciones contingentes sobre el patrimonio neto de la empresa, tales como la existencia de obligaciones convertibles en acciones u opciones sobre acciones (stock options) de los ejecutivos. Asimismo, afirma que:

El estado de resultados 'completo' — globalototal según nuestra denominacióno un enfoque equivalente que provee el 'excedente limpio', en la medida en que la suma total de las ganancias anuales, desde el inicio de una compañía (que se inicia con caja) hasta su finalización (terminando también con caja), es igual a, precisamente, la suma total de las ganancias anuales de toda la vida de la compañía (2002: 50) .

\footnotetext{
${ }^{1}$ Para profundizar en el sustento teórico del resultado global pueden consultarse, entre otros, Linsmeier et al., 1997; Gonzalo, 2000; Beale y Davey, 2000; Mattessich, 2002 y Sousa, 2007.
} 
Por todo ello, con la adopción del resultado global nos hallamos ante un claro acercamiento al concepto de resultado económico, defendido por los autores de la escuela clásica normativo deductiva² (MacNeal, 1939; Alexander, 1950; Edwards y Bell, 1961; Moonitz, 1961; Sprouse y Moonitz, 1962, entre otros). Pero en la actualidad no es concebido como una magnitud a priori única e incontestable, sino pensado para satisfacer las necesidades de los usuarios, en particular las de los inversionistas, dado que con el mismo se contribuye al funcionamiento eficiente del mercado y a la utilidad de la información contable para la valoración de las empresas en los mercados (Mora, 2004: 10).

En contraposición a estas concepciones, tenemos el resultado realizado tradicional, también conocido como excedente sucio, del que hemos extraído esta definición de Martínez y Sousa:

La diferencia entre los ingresos realizados derivados de las transacciones del periodo y los gastos asociados a los mismos, valorados conforme a su costo histórico y con una aplicación de la prudencia orientada a la sobrevaloración consciente de pasivos y gastos y la minusvaloración deliberada de activos e ingresos (2004: 4).

Desde esta perspectiva, coincidente con los planteamientos de los autores de la escuela positivo inductiva (Hatfield, 1927; Sanders, Hatfield y Moore, 1938; Gilman, 1939; Ijiri, 1975, entre otros), resulta obvio que el resultado contable no captura todos los cambios en la riqueza de la empresa para la propiedad porque algunos de ellos quedan relegados a la "oscuridad" al ser reconocidos directamente en cuentas de neto o en la memoria.

Así, desde estos planteamientos, el resultado empresarial queda confundido o disperso entre el estado de resultados tradicional, el balance o las notas explicativas, lo que conduce a que la suma de los resultados revelados anualmente no coincida con el resultado total acumulado a lo largo de la vida de la empresa. Precisamente, por estos motivos recibe en la literatura y la regulación contables anglosajonas el calificativo peyorativo de "sucio".

${ }^{2}$ Para profundizar en la contabilidad como disciplina multiparadigmática se puede consultar, entre otros, AAA (1977); Túa (1983); Cañibano y Gonzalo (1997) y Belkaoui (2004). 
Pero Mattessich en relación con las anteriores consideraciones afirma que "esto no implica que los estados de resultados sucios no sean respetables" (2002: 50-51); por ello, argumenta a favor del enfoque del excedente sucio en el sentido de que normaliza las ganancias de la empresa entre varios periodos de cara a mostrar la tendencia a largo plazo de las mismas, en lugar de mostrar una serie de cifras de beneficios anuales fluctuantes y posiblemente erráticas.

En definitiva, y tal como se detalla a continuación, nos encontramos ante dos concepciones enfrentadas con respecto a la determinación del resultado empresarial: una globalizadora, que en su concepción más genuina, incluye todos los cambios en el valor de activos y pasivos que afectan al neto excluyendo operaciones con los propietarios; otra más restrictiva, que se limita a captar los resultados realizados tradicionales basados esencialmente en las transacciones que lleva a cabo la empresa.

\section{El debate resultado globalizador -all inclusive concept of income- (o del excedente limpio) versus resultado corriente operativo -current operating concept of income - (o del excedente sucio)}

La historia del pensamiento contable no presenta una continuidad evolutiva, sino más bien una serie de episodios discontinuos (Chambers, 1965: 33); por ello, es necesario enfatizar que si la evolución se hubiera llevado a cabo de una forma ordenada, sistemática y disciplinada, ninguna nueva regla habría sido admitida a no ser que fuese más aceptable que otra ya existente para conseguir un determinado propósito, lo que llevaría aparejado la eliminación inmediata de la regla o reglas sustituidas.

Las razones de esta falta de continuidad en la evolución del pensamiento contable radican en buena parte en que los conceptos contables, o los fenómenos a los que van referidos los mismos, no pueden ser interpretados únicamente desde la estricta objetividad y de forma mecánica. En concreto, el resultado empresarial que nos ocupa, aún representando una de las variables fundamentales de la información financiera, es una magnitud que ni es objetiva ni es observable por definición (Gonzalo, 1996: 573). ${ }^{3}$

\footnotetext{
${ }^{3}$ Puede consultarse el trabajo de Lukka (1990) en el que se estudia el concepto de resultado contable en el marco de las ontologías realista e idealista.
} 
Estas consideraciones sirven de apoyo en el discurrir del controvertido debate del enfoque del excedente limpio versus enfoque del excedente sucio en la determinación y presentación del resultado contable, que en algunas partes de su revisión nos basaremos esencialmente en la antología de Brief y Peasnell (1996), así como en los trabajos de Beale y Davey (2000) y Mattessich (2002), donde existen enfoques y reglas enfrentadas, representando en muchos casos una secuencia de trayectos interrumpidos, tal como hemos referido.

En este sentido, al analizar el significado del estado de resultados en Gran Bretaña en el siglo XIX, Jones y Aiken (1994) revelan dos conceptos de resultado presentes en el marco de la regulación contable: uno basado en el método del excedente y el otro en el método del estado de resultados. El primero de ellos se fundamenta en el resultado global, con lo que tanto los beneficios como las pérdidas no realizadas quedan integrados en el mismo, entre las que destacan las derivadas de una revalorización de activos fijos. En cuanto al segundo método, el resultado se determina por la diferencia entre los ingresos y los gastos asociados al periodo adecuadamente imputados, de tal modo que otros cambios en el valor de los activos y pasivos no tendrían cabida.

No obstante lo anterior, tal como ponen de manifiesto Beale y Davey (2000: 8283), existen posturas contradictorias en la literatura anglosajona con respecto al grado de aceptación del resultado global en el siglo XIX. Por su parte, Kam señala que "durante el siglo XIX, el resultado de una empresa de negocios se determinaba sobre la base de un incremento en la riqueza neta" (1986: 177), para lo cual se basa, entre otras, en las afirmaciones de May: "Una revisión de los trabajos en el ámbito contable, legal y económico sugiere que el postulado de la realización no fue aceptado antes de la Primera Guerra Mundial. En 1913, autoridades destacadas en estos ámbitos en Inglaterra ( ic ) y América ( sic) parecen haber estado de acuerdo con el concepto de beneficio como 'el incremento en la riqueza neta' [...]" (1950: 316).

Sin embargo, Jones y Aiken cuestionan esta preponderancia del resultado global al considerar que aunque tanto el método del excedente como el método del estado de resultados hayan jugado un importante papel en la determinación del beneficio empresarial en el siglo XIX en Gran Bretaña los profesionales y las opiniones legales en las últimas décadas de ese siglo parecen haber favorecido este último método (1994: 203-204). 
Este debate, que gira en torno al principio de realización versus resultado global, continúa al inicio del siglo XX. Así, Brief y Peasnell (1996: 7) revelan que Dickinson (1908: 208) distinguía entre un estado de resultados preparado para presentar a su aprobación a la junta general de accionistas y otro elaborado a modo de folleto informativo con el propósito de comunicar información a los principales inversionistas sobre la capacidad de la empresa para la generación de resultados futuros, en la que quedarían excluidos elementos relacionados con cambios en las bases de valoración.

Después de la Primera Guerra Mundial, el resultado determinado conforme al incremento en la riqueza neta fue perdiendo posiciones a favor del resultado calculado bajo el principio de realización. Kam señala que las razones de esta inclinación a favor del resultado realizado es el hecho de que las empresas empezaban a invertir en activos físicos muy especializados, para los cuales se hacía muy difícil encontrar criterios de valoración adecuados, así como a la circunstancia que las leyes fiscales tanto en Gran Bretaña como en Estados Unidos se manifestaban en contra de reconocer los ingresos como un incremento en la riqueza neta (1986: 177-178).

Tal como refieren Kam (1986), Bealey y Davey (2000) y Mattessich (2002), entre otros, la polémica llegó a trascender hasta los tribunales de justicia. Así, del siguiente extracto de la sentencia del Tribunal Supremo en el caso Eisner vs Macomber [25 USA 188,195], fechada en 1920, se deduce claramente la opción del tribunal por un concepto de resultado que incluya únicamente beneficios y pérdidas realizadas:

Está en la propia esencia del resultado que éste debe ser realizado. La potencialidad no es suficiente [...] El resultado implica necesariamente separación y realización. El incremento de los bosques no es resultado hasta que no se hayan talado. El incremento en el valor de los terrenos debido al incremento de la prosperidad en la comunidad no es resultado hasta que no esté realizado. En el caso de que estén implicadas inversiones, no hay resultado hasta que no haya un separado, beneficio realizado [...].

Paton y Stevenson (1916: 183) y Paton (1922: 187) abogaban por reconocer en el estado de resultados pérdidas y ganancias que no tuvieran ninguna relación con las operaciones ordinarias de la empresa, entre las que se citan la apreciación en el valor de un terreno o las pérdidas ocasionadas en los activos por una inundación. 
Posteriormente, con el objeto de prevenir una incorrecta presentación del resultado, Paton (1934: 128) establece que deben tomarse en cuenta todas las partidas recogidas en cuentas de reservas que pudieran incluir algún resultado del ejercicio, por ello se incorporaron al estado de pérdidas y ganancias.

De igual modo, Brief y Peasnell (1996: xiii) constatan que en el modelo de estado de resultados propuesto por el Federal Reserve Board, en su Federal Reserve Bulletin del 1 de abril de1917, se hacía referencia a un concepto global de resultado, pero posteriormente en su revisión, realizada por el American Institute of Accountants (Federal Reserve Board, 1929), fue modificado al adoptarse el enfoque del excedente sucio.

Pero la Gran Depresión de 1929 fue el elemento detonante que hizo tomar posiciones a favor del resultado realizado tradicional, debido a que - como considera Kam (1986: 179) — los abusos en las valoraciones registrados en la década de los veinte del siglo XX contribuyeron en parte al desastre económico hasta el extremo de afirmar que "algunos vieron a la profesión contable, en parte, responsable de los calamitosos acontecimientos, al haber permitido a las compañías valorar activos de forma muy optimista".

En este sentido, Beale y Davey (2000: 84) —aparte de señalar el incremento en el uso de activos especializados y las normas fiscales como factores que provocaron un giro a favor del resultado determinado bajo el imperio del principio de realización, tal como hemos referido- consideran también como hecho determinante de este giro "las lecciones aprendidas de la Gran Depresión", cuando se refieren a estas afirmaciones de Chatfield: "En relación con estas críticas [valoración de activos de forma muy optimista], los contables adoptaron una actitud conservadora, y el principio de reconocimiento [realización] fue un resultado de su postura defensiva" (1977: 260).

Años más tarde, después de los sucesos de la Gran Depresión, se publica el trabajo de Gilman (1939) que representa un claro exponente de las posiciones a favor del principio de realización. En el mismo se revisan distintos conceptos de resultado y se referencia a distintos autores (1939: 231), entre otros, a Sanders, Hatfield y Moore), que son claramente partidarios del resultado realizado, como lo prueban estas afirmaciones suyas: "Únicamente el resultado realizado por la venta de las existencias o la prestación de servicios ha de incluirse en el estado de resultados. Los beneficios no realizados no deberían ser registrados, ni utilizados para absorber las adecuadas cargas contra el beneficio" (1938: 114). 
Sin embargo, prácticamente coincidente en el tiempo con los anteriores autores, el Executive Committee de la American Accounting Associtation (1936: 189-190) se alinea con el enfoque del excedente limpio al establecer que:

El estado de resultados de un determinado periodo debería reflejar todos los ingresos adecuadamente reconocidos y todos los costos incurridos, con independencia de si son resultados de las operaciones del periodo, de tal modo que para cualquier conjunto de periodos en la historia de la empresa la acumulación de los estados de resultados exprese completamente todos los gastos y los ingresos.

Para ello, proponen estructurar el estado de resultados en dos agrupaciones principales: resultados operativos y resultados de capital, integrando en esta última categoría beneficios y pérdidas extraordinarios no relacionados con las operaciones, incluidos los correspondientes a ejercicios anteriores, beneficios y pérdidas en la liquidación de deudas a un valor superior o inferior al reconocido, y otros ítems similares.

Es importante destacar de ese documento lo siguiente:

Los estados de resultados para una serie de ejercicios no deberían ser tergiversados o estabilizados artificialmente a través de prácticas consistentes en dotar grandes reservas en unos periodos y cargar contra esas reservas pérdidas de los periodos subsiguientes, las cuales no se desean reflejar en resultados del periodo corriente [...] Cualquier pérdida asociada a contingencias debería ser reflejada, no en reservas, sino en el estado de resultados del periodo en que se incurre en la misma (p.190).

Del mismo modo, otros autores como MacNeal o Alexander son claros defensores del excedente limpio, el primero por considerar "un verdadero estado de resultados" que incluya en un epígrafe independiente todos los beneficios y pérdidas de capital, estén realizados o no (1939: 194); y el segundo al declararse partidario a lo largo de su trabajo del concepto económico de resultado frente al beneficio realizado tradicional, argumentando esencialmente que "es mejor actuar evaluando hechos subjetivos de factores relevantes que de una forma perfectamente objetiva pero valorando acontecimientos irrelevantes" (1950: 60).

Asimismo, el concepto de resultado global estaba presente de forma explícita en Littleton al proponer un formato de estado de resultados en el que quedasen inte- 
grados, por una parte, los resultados recurrentes (operativos y no operativos) y, por otra, los resultados no recurrentes (operativos y no operativos) (1940: 37-38). Cabe señalar que en el epígrafe referente a los resultados derivados de las transacciones no recurrentes se incluyen tanto beneficios como pérdidas realizados y en potencia. ${ }^{4}$

Por su parte, Paton y Littleton, aún sin referirse expresamente al resultado global, tienen implícito el concepto al establecer que "todos los elementos determinantes del resultado contable en el más amplio sentido - incluyendo factores inusuales e irregulares - deberían ser reconocidos en el estado de pérdidas y ganancias antes de proceder a su traslado a los fondos propios" (1940: 102). Asimismo, estos autores señalan que "aunque el costo histórico ha de ser la base sobre la cual se han de formular los estados financieros, sería razonable el suministro de información complementaria que reflejase las variaciones en los precios, siempre que reportase claramente utilidad" (1940: 126). Así, para completar la información financiera contenida en los epígrafes tradicionales, proponen como forma de representación de esos cambios en los valores corrientes de los elementos de los estados financieros, la inclusión de notas en la memoria, columnas extra, etc., para lo cual afirman que "la contabilidad no debería tener límites de cara a suministrar esa información pertinente" (1940: 126).

Sin embargo, de nuevo volvemos a encontrar planteamientos contrarios al clean surplus. Como señalan Brief y Peasnell (1996: xiv), que a su vez retoman a Zeff (1972: 157), las posturas enfrentadas durante largo tiempo entre la Securities and Exchange Commission (SEC) y el American Institute of Accountants (AIA) llegan al punto de máximo desacuerdo en 1947 con la publicación del Accounting Reseach Bulletin $\mathrm{N}^{\mathrm{o}} 32$. Mientras la SEC había expresado su preferencia por un estado de resultados globales, el AIA abogaba por un concepto de resultado fundamentado en operaciones de naturaleza corriente.

El periodo que va desde 1941 a 1973, según Kiger y Williams (1977: 63), se caracterizó por la adopción de posturas eclécticas, llegando a un modelo híbrido entre el resultado global y el resultado operativo. A mediados de la década de los setenta, el Financial Accounting Standards Board (FASB) en el Statement of Financial

\footnotetext{
${ }^{4}$ Téngase en cuenta que en el epígrafe correspondiente a los beneficios no realizados aparecen con un interrogante (unrealised gains?), lo que significa la posibilidad de incluir también los beneficios en potencia dentro del estado de resultados
} 
Accounting Standards (SFAS 8) (1975): "Accounting for the Traslation of Foreign Currency Transactions and Foreign Financial Statements", se adhiere al concepto de resultado global, como queda de manifiesto en el párrafo 183 al establecer:

Se recibieron sugerencias en el periodo de discusión del borrador de la norma con respecto a que las diferencias de cambio en moneda extranjera se reconociesen directamente en el patrimonio neto. El Board rechazó tal método al estimar que los beneficios y las pérdidas derivados de la exposición al riesgo de cambio, de acuerdo con principios de contabilidad generalmente aceptados, deberían ser incluidas dentro del Estado de Resultados, adoptándose así el enfoque clean surplus. Una inversión en moneda extranjera expone a las empresas estadounidenses a los efectos del cambio de la moneda que pueden ser beneficiosos o perjudiciales. El Board cree que esos beneficios y pérdidas deberían ser reflejados en la determinación del resultado, en tiempo y forma, y de acuerdo con los principios de contabilidad generalmente aceptados.

No obstante, el FASB reconoció que la aceptación del concepto de resultado global incrementaría la volatilidad del resultado contable; sin embargo, argumentó que "si los tipos de cambio fluctúan, la contabilidad no debe dar una impresión de tipos de cambio estables” (párrafo 199).

Ahora bien, como señala Walsh (1995: 2-3), la situación descrita duró poco tiempo debido a que el FASB cedió ante el elevado número de presiones en relación con los métodos de conversión y de reconocimiento de gastos e ingresos y reconsideró el SFAS 8, que fue reemplazado por el SFAS 52 "Foreign Currency Translations", emitido en 1981. En esa nueva norma se daba la posibilidad a las empresas de reconocer dentro de una rúbrica independiente en el patrimonio neto los ajustes de conversión de moneda extranjera, tanto positivos como negativos, eludiendo con ello su representación en el estado de resultados.

Este giro del Board desde un enfoque clean surplus a otro dirty surplus se acentúa años más tarde con la emisión de las normas SFAS 87 (1985): "Employees Accounting for Pensions" y sFas 115 (1993): "Accounting for Certain Investments in Debt and Equity Securities", que obligaban a reconocer directamente en el patrimonio neto tanto las variaciones en la provisión del fondo de pensiones como los beneficios y pérdidas no realizados en inversiones financieras, respectivamente. 
Por otra parte, en este debate, Cea señala:

La existencia de operaciones potenciales o sucesos aleatorios en la fecha de cierre del ejercicio y, por tanto, en la fecha de cálculo del resultado contable no debería alterar la cifra de resultados del ejercicio, la cual para ser plenamente expresiva del auténtico excedente económico del ejercicio transcurrido debería circunscribirse exclusivamente a las operaciones lucrativas devengadas dentro del mismo. Las ganancias y, sobre todo, las pérdidas potenciales o aleatorias afectan sobre todo al ambiente de riesgo que rodea el resultado de las operaciones devengadas y a la magnitud de los recursos propios con que cuenta la empresa como expresión contable de su garantía frente a sus acreedores, por lo que deberían tener cobertura informativa (en el Balance - provisiones con cargo a partida específica minorada de los recursos propios- o en la Memoria) y, en su caso, la cifra acumulada de esta clase de pérdidas debería actuar como restricción del reparto como dividendos del resultado contable computado o del reparto de las reservas (1994: 57).

Tales planteamientos, por las razones señaladas, quedan encuadrados dentro del enfoque dirty surplus. Una década después, Cea continúa con un planteamiento similar al considerar que "el resultado contable de un periodo correspondiente a las operaciones devengadas debe ser el correspondiente a las operaciones concluidas, es decir, resultado maduro (realizado) y no el de las operaciones no concluidas o resultado todavía prematuro (no realizado)" (2005:142); esto es, incorpora un importante matiz inclinado a favor del enfoque clean surplus.

Así, considera que los activos de naturaleza extrafuncional o especulativa y destinados a ser vendidos en su forma actual — si cotizan en mercados organizados y abiertos con precios o cotizaciones transparentes, con liquidación garantizada y sin que la empresa con esos elementos pueda incidir decisivamente en los precios de mercado- deberían valorarse continuamente a su cotización neta de mercado, reconociendo las diferencias de valoración detectadas como componentes del resultado contable periódico, por ser devengado y maduro en términos económi$\cos (2005: 147)$.

Después de efectuar la revisión sobre el debate del enfoque del excedente sucio versus enfoque del excedente limpio, lejos de darse un acuerdo general con respecto al cálculo y la presentación del resultado contable, podemos percibir posturas enfrentadas desde el siglo XIX hasta la actualidad, las cuales pueden quedar sinte- 
tizadas en dos enfoques esencialmente incompatibles con respecto a la configuración del resultado empresarial:

- "El resultado es el incremento en la riqueza de la empresa" (Storey y Storey, 1998: 76). Basado en los cambios de valor de activos y pasivos que afecten al patrimonio neto, excepto operaciones con los propietarios (Hendriksen, 1982: 137-171).

- "El resultado es el indicador del rendimiento -performance- de la empresa y de su management" (Storey y Storey, 1998: 76). Basado en las transacciones y en el principio de realización bajo el modelo del costo histórico (Hendriksen, 1982: 137-171).

Como se analiza posteriormente al referirnos a la NIC 1 del IASB revisada en 2007, en la actualidad nos hallamos ante un posicionamiento a favor del enfoque del excedente limpio, de tal modo que en su concepción más genuina el resultado empresarial captura todos los acontecimientos relevantes desde el punto de vista del valor, lo que nos lleva a concebir el mismo como la variación en la riqueza neta de la empresa una vez excluidas las operaciones con la propiedad.

Las razones que definen esta tendencia - concretada ya en las correspondientes normas reguladoras del resultado global a partir la década de los noventa del siglo $\mathrm{XX}$, entre las que cabe destacar el FRS 3 del ASB, el SFAS 130 del FASB y la NIC 1 del IASB - pueden quedar sintetizadas esencialmente en las siguientes:

- La emisión del marco conceptual del FASB en pleno desarrollo del paradigma de utilidad, que propugna como características primordiales de la información financiera la relevancia y fiabilidad y reconoce como usuarios de referencia a los inversionistas.

- En coherencia con los anteriores planteamientos, y tal como se ha señalado, se concibe el resultado contable como una variación en la riqueza neta eliminando operaciones con los propietarios; esta concepción es secundada después por los organismos emisores de normas contables más relevantes en el panorama internacional.

- El fuerte desarrollo de los instrumentos financieros y derivados desde la década de los noventa del siglo XX, junto con la irrupción del valor razonable para la valoración de un número significativo de ellos, que motiva el debate sobre el traslado a resultados de las diferencias registradas en el mismo, bien 
al estado de resultados, o al patrimonio neto para ser integradas después en el resultado global o excedente limpio.

- Las demandas de los analistas e inversionistas, entre los que cabe destacar la posición de la AIMR (1993), en la actualidad el Chartered Financial Analyst Institute (CFA Institute), asociación líder de los analistas financieros en Estados Unidos, al propugnar la revelación del resultado global en el cuerpo principal de los estados financieros, aduciendo como motivo esencial el incremento de la relevancia para los suministradores de capital-riesgo, que según hemos visto son considerados en el marco conceptual como usuarios de referencia.

Sin embargo, dada la gravedad de la actual crisis, estamos asistiendo de nuevo a un controvertido debate con respecto al empleo de valores de mercado, o markto-marked accounting en terminología anglosajona, para la valoración de determinados instrumentos financieros con las correspondientes consecuencias en la determinación del resultado empresarial.

En este sentido, en el informe sobre el uso del mark-to-marked accouting que la U. S. Securities and Exchange Commission (SEC, 2008) ${ }^{5}$ ha remitido al Congreso de los Estados Unidos en el marco del "Plan de emergencia para la estabilización económica" ante la grave crisis; además de reflejar los argumentos de los detractores y defensores del mark-to-market accounting, la SEC no es partidaria de suspender las valoraciones contables conforme a dicho criterio, pero sí recomienda la mejora de determinadas normas que lo regulan.

Por una parte estamos ante los detractores del mark-to-market accounting o de la contabilidad a valor razonable —en particular las entidades financieras integradas en la American Bankers Association (ABA) — dado que consideran que el mismo ha provocado inestabilidad en los mercados financieros y, por ello, es el responsable en buena medida de la actual crisis económica. Argumentan que al tener que aplicar el valor razonable, principalmente cuando los mercados son ilíquidos o inactivos, les ha llevado a valorar determinadas inversiones financieras muy por debajo de su "valor económico verdadero". Esto ha conducido, por parte de muchas entidades financieras, al incumplimiento de los requisitos regulatorios de capital, y

\footnotetext{
${ }^{5}$ Por razones de espacio limitado no se aborda de forma exhaustiva la problemática contenida en dicho informe, pero dada la naturaleza de los contenidos tratados, en línea con nuestro trabajo de investigación, puede resultar de mucho interés su consulta.
} 
al no contar con suficientes recursos para poder compensar las fuertes pérdidas por valor razonable que les permitieran el cumplimiento con dichos requerimientos de capital se han visto abocadas a la quiebra.

Por otra parte, estamos ante los defensores del mark-to-market accounting porque consideran que el mismo refuerza la transparencia de la información financiera revelada a los usuarios, y que la eliminación de las valoraciones según dicho criterio supondría debilitar la confianza de los inversionistas con la consiguiente inestabilidad en los mercados. Asimismo, estiman que las decisiones de crédito equivocadas, una inadecuada gestión del riesgo con la consiguiente generación de activos tóxicos y las debilidades inherentes de la supervisión y regulación son los factores que esencialmente motivaron la grave crisis económica actual, y no las valoraciones conforme al mark-to-marked accounting.

En el marco de este controvertido debate, en el citado informe de la SEC (2008), se analizan, entre otros aspectos, los efectos del valor razonable en los balances de las entidades financieras estadounidenses, el impacto de este criterio valorativo en las quiebras bancarias del 2008 en Estados Unidos y en la calidad de la información financiera suministrada a los inversionistas, así como las alternativas disponibles en la normativa y la conveniencia de reforma de las normas que lo regulan.

En ese sentido, se considera que el valor razonable no ha sido determinante en las quiebras bancarias del 2008 y que su suspensión a favor del costo histórico incrementaría la incertidumbre de los inversionistas.

Por ello, entre las recomendaciones contenidas en el informe de la SEC, cabe destacar el hecho de que deben mantenerse las valoraciones conforme al valor razonable y al mark-to-marked accounting contempladas en la normativa vigente. No obstante, se considera necesario mejorar dicha normativa, particularmente en lo que respecta al tratamiento de los deterioros de valor, la aplicación práctica del valor razonable contemplada en el SFAS 157 (2006) del FASB, el valor razonable de los pasivos y la presentación de la información a los usuarios en el marco del proyecto conjunto que están desarrollando el FASB (2009) y el IASB (2009). ${ }^{6}$

\footnotetext{
${ }^{6}$ Algunas de estas mejoras ya han sido emprendidas y otras están en curso en la actualidad. Puede consultarse al respecto en la web del FASB: http://www.fasb.org, los Final FASB Staff Positions: FSP SFAS 107-1 y APB 28-1, FSP SFAS 115-2 y SFAS 124-2 y FSP SFAS 157-4.
} 
En definitiva, como ya se indicó, la Gran Depresión de 1929 provocó el retorno en aquel entonces a las valoraciones conforme al costo histórico y, con ello, a la configuración de un estado de resultados según los planteamientos conservadores del enfoque del excedente sucio.

Sin embargo, ante la grave crisis actual, aunque existan partidarios de eliminar las valoraciones de los activos y pasivos conforme al mark-to-market accounting, así como el correspondiente impacto en el estado de resultados, la voluntad de seguir manteniendo dicho criterio por parte de la SEC y del FASB e IASB, evidentemente con las mejoras que ya se están teniendo en cuenta en el mismo con su aplicación práctica, confirman ahora a inicios del siglo XXI —al menos desde la óptica de los reguladores - un posicionamiento a favor de enfoque del excedente limpio, motivado esencialmente por el suministro de una información relevante a los inversionistas.

\section{El declive del estado de resultados tradicional ante la irrupción del resultado global o un enfoque equivalente que provee el excedente limpio}

Como ya se mencionó, a partir de la década de los noventa del siglo XX los organismos emisores de normas contables más representativos, entre los que cabe señalar el ASB, el IASB y el FASB, tomaron la decisión de incorporar en resultados determinados elementos que se reconocen en el patrimonio neto, lo que una vez agregados al resultado neto (net income) se llega a la última magnitud resultado global (comprehensive income). Esto permitió que determinadas partidas con tratamiento excedente sucio - - y que por lo tanto no formaban parte del resultado del ejercicio, sino que se reconocían en el patrimonio neto, tales como determinadas diferencias de conversión en moneda extranjera, los cambios en el valor razonable de instrumentos financieros clasificados como disponibles para la venta o las pérdidas y ganancias asociadas a coberturas de flujos de efectivo- fuesen incorporadas al resultado global con lo que así se adopta el enfoque del excedente limpio.

Para ello, las empresas han venido presentando el resultado global teniendo en cuenta distintas opciones, entre las que interesa destacar la posibilidad de hacerlo en el estado de cambios en el patrimonio neto o en el estado de ingresos y gastos reconocidos, según la NIC 1 (2003). Sin embargo, con la revisión de dicha norma en 2007, que entra en vigor el primero de enero de 2009, el IASB contempla la revelación obligatoria del resultado global en el estado de resultados globales (Sta- 
tement of Comprehensive Income), según se recoge en el cuadro 1, considerado como un estado contable con autonomía propia dentro del conjunto completo de estados financieros, lo que supone un mayor afianzamiento del enfoque del excedente limpio.

Asimismo, las empresas podrán optar por presentar el estado de resultados tradicional de forma separada, que según podemos observar abarca hasta el "beneficio del periodo" junto con la atribución del beneficio y el beneficio por acción; e inmediatamente después el estado de resultados globales, elaborado a partir del saldo del primero, que lo integra; o bien, presentar sólo este último estado, con lo que en este caso estaríamos ante un destacado hecho para la contabilidad que consistiría en la desaparición del estado de resultados tradicional del conjunto completo de estados financieros.

Tal como se contempla en el párrafo IN1 de la NIC 1 (2007), la principal razón que ha motivado la revisión de la misma ha sido la de agregar en los estados financieros información con características comunes, particularmente en lo concerniente a los cambios en el patrimonio neto. ${ }^{7}$

De este modo, han de diferenciarse nítidamente todos los cambios no relacionados con operaciones con la propiedad, que son precisamente aquéllos que inicialmente definen el beneficio del periodo junto con los que después conforman el resultado global (enfoque del excedente limpio), tal como quedan recogidos en el cuadro 1; aquellos otros que guardan relación con los propietarios como pueden ser las ampliaciones o reducciones de capital, la conversión de obligaciones en acciones o el reparto de dividendos se informarán de ellos en el estado de cambios en el patrimonio neto.

\footnotetext{
${ }^{7}$ Como quedó señalado, a partir de la entrada en vigor de dicha norma el primero de enero de 2009 , ya no se podrá optar por revelar el resultado global en el estado de cambios en el patrimonio neto, destinándose el mismo a informar de los cambios en el patrimonio relacionados con operaciones con la propiedad, junto con una mera síntesis de los no asociados a operaciones con los propietarios, que son los que conforman el resultado global.
} 


\section{Cuadro 1 \\ Estado de Resultados Globales según la NIC 1 del IASB revisada en $2007^{8}$ (Statement of Comprehensive Income) [Asunción del enfoque clean surplus]}

\begin{tabular}{|c|c|c|}
\hline & $200 \mathrm{X}$ & $200 X-1$ \\
\hline Ingresos & $\mathrm{X}$ & $\mathrm{X}$ \\
\hline Otros ingresos & $\mathrm{X}$ & $\mathrm{X}$ \\
\hline Variación de existencias de productos terminados y trabajos en curso & $(\mathrm{X})$ & $(\mathrm{X})$ \\
\hline Trabajos llevados a cabo por la empresa y capitalizados & $\mathrm{X}$ & $\mathrm{X}$ \\
\hline Materias primas y consumibles utilizados & $(\mathrm{X})$ & $(\mathrm{X})$ \\
\hline Gastos de personal & $(\mathrm{X})$ & $(\mathrm{X})$ \\
\hline Amortizaciones & $(\mathrm{X})$ & $(\mathrm{X})$ \\
\hline Deterioro del inmovilizado & $(\mathrm{X})$ & $(\mathrm{X})$ \\
\hline Otros gastos & $(\mathrm{X})$ & $(\mathrm{X})$ \\
\hline Costos financieros & $(\mathrm{X})$ & $(\mathrm{X})$ \\
\hline Participación en el beneficio de las asociadas ${ }^{1}$ & $\mathrm{X}$ & $\mathrm{X}$ \\
\hline Beneficio antes de impuestos & $\mathrm{X}$ & $\mathrm{X}$ \\
\hline Impuesto sobre beneficios & $(\mathrm{X})$ & $(\mathrm{X})$ \\
\hline Beneficios del periodo de operaciones continuadas & $\mathrm{X}$ & $\mathrm{X}$ \\
\hline Pérdidas del periodo de operaciones interrumpidas & - & $(\mathrm{X})$ \\
\hline BENEFICIO DEL PERIODO & $\mathrm{X}$ & $\mathrm{X}$ \\
\hline Otros elementos del resultado global & & \\
\hline $\begin{array}{l}\text { Diferencias de cambio en la conversión de operaciones } \\
\text { extranjeras }\end{array}$ & $\mathrm{X}$ & $\mathrm{X}$ \\
\hline Activos financieros disponibles para la venta & $(\mathrm{X})$ & $\mathrm{X}$ \\
\hline Coberturas de flujos de caja & $\mathrm{X}$ & $\mathrm{X}$ \\
\hline Beneficios en la revalorización del inmovilizado material & $\mathrm{X}$ & $\mathrm{X}$ \\
\hline $\begin{array}{l}\text { Beneficios (pérdidas) actuariales en planes de pensiones } \\
\text { de prestaciones definidas }\end{array}$ & (X) & (X) \\
\hline $\begin{array}{l}\text { Participación de las asociadas en otros elementos del } \\
\text { resultado global }\end{array}$ & $\mathrm{X}$ & $(\mathrm{X})$ \\
\hline $\begin{array}{l}\text { Impuesto sobre beneficios correspondiente a otros } \\
\text { elementos del resultado global }\end{array}$ & $\mathrm{X}$ & $(\mathrm{X})$ \\
\hline Otros elementos del resultado global, netos de impuestos $\quad$ & $(\mathrm{X})$ & $\mathrm{X}$ \\
\hline TOTAL RESULTADO GLOBAL (COMPREHENSIVE INCOME) DEL PERIODO & $\mathrm{X}$ & $\mathrm{X}$ \\
\hline \multicolumn{3}{|l|}{\begin{tabular}{|l|l|} 
Beneficio atribuible a: & \\
\end{tabular}} \\
\hline - Propietarios de la matriz & $\mathrm{X}$ & $\mathrm{X}$ \\
\hline \multirow[t]{2}{*}{ - Intereses sin control } & $\mathrm{X}$ & $\mathrm{X}$ \\
\hline & $\mathrm{X}$ & $\mathrm{X}$ \\
\hline \multicolumn{3}{|l|}{ Total resultado global (comprehensive income) atribuible a: } \\
\hline - Propietarios de la matriz & $\mathrm{X}$ & $\mathrm{X}$ \\
\hline - Intereses sin control & $\mathrm{X}$ & $\mathrm{X}$ \\
\hline & $\mathrm{X}$ & $\mathrm{X}$ \\
\hline \multicolumn{3}{|l|}{ Beneficio por acción: } \\
\hline - Básico y diluido & $\mathrm{X}$ & $\mathrm{X}$ \\
\hline
\end{tabular}


Fuente: elaboración y traducción propias a partir de la Guía para la Implementación de la NIC 1 (2007), Parte I, en Bound Volume of International Financial Reporting Standards (IFRSs ®), 2008, IASB, IASC Foundation, London.

Por último, interesa resaltar el debate sobre la presentación del rendimiento empresarial $^{9}$ recogido en la sección de Bases para las conclusiones de la referida NIC, específicamente, en los apartados BC49 a BC54 relacionados con el párrafo 81 de la misma. Así, el IASB, a pesar de haber mantenido en dicha norma la posibilidad de seguir formulando un estado de resultados tradicional de forma separada debido a las sugerencias y la polémica suscitada en el periodo de su discusión, se declara partidario de presentar el resultado global en un solo estado, esto es, en el estado de resultados globales.

Para lo anterior aduce como razón esencial que si en el marco conceptual se definen los ingresos y los gastos como integradores todos ellos de los cambios en el patrimonio neto no relacionados con operaciones con la propiedad, no hay motivos que puedan justificar su desglose en dos estados contables diferentes.

Este posicionamiento del IASB, que evidentemente supone la no consideración del estado de resultados tradicional al quedar subsumido en el estado de resultados globales, previsiblemente prevalecerá en un futuro a medida que vaya avanzando el proyecto "Presentación de estados financieros", que está desarrollando conjuntamente con el FASB.

De confirmarse esta postura del IASB, supondría a medio o largo plazo la desaparición ya de forma definitiva de los estados de resultados tradiciones del conjunto completo de estados financieros periódicos de propósito general; con ello, a inicios del siglo XXI, asistiríamos al alejamiento de forma consolidada de los planteamientos propugnados por los defensores del enfoque del excedente sucio.

\section{Conclusiones}

Nos hemos adentrado en el controvertido debate en torno al enfoque del excedente sucio versus enfoque del excedente limpio en la determinación y presentación del

\footnotetext{
${ }^{8}$ Se muestra el estado formulado adoptando una clasificación por naturaleza de los gastos asociados al beneficio del periodo, pero las empresas también podrán optar por una clasificación funcional de los mismos, lo que en este último caso requerirá explicar en las notas su composición por naturaleza.

${ }^{9}$ Puede también consultarse al respecto el documento del EFRAG-ICAC (2006).
} 
resultado empresarial. Esta confrontación se encuentra esencialmente en el ámbito de la literatura y regulación contables anglosajonas, que se inicia en el siglo XIX, se intensifica en el siglo XX y continúa en este siglo XXI. Se detecta la ausencia de una continuidad evolutiva en el desarrollo del referido debate, en el sentido de que hemos constatado la preeminencia del enfoque del excedente limpio en unas etapas concretas y la supremacía del enfoque del excedente sucio en otras.

Esto se debe principalmente al hecho de que en el marco de la contabilidad como disciplina multiparadigmática, el resultado empresarial —al igual que el resto de conceptos contables - no representa categorías absolutas que puedan ser concebidas desde la estricta objetividad. De ahí que se deriven criterios, reglas o convenios muchas veces enfrentados tanto en la literatura como en la regulación contables, que particularmente han dificultado el discurrir del debate con respecto a la determinación y presentación del resultado empresarial de una forma ordenada y sistemática.

En este sentido, la problemática de fondo que se presenta al revisar ambos enfoques gira en torno a sendos planteamientos esencialmente incompatibles con respecto a la configuración del resultado empresarial.

De este modo, conforme al enfoque del excedente limpio, el resultado empresarial se concibe como la variación en la riqueza de la empresa, basado en los cambios de valor en las valoraciones autónomas de activos y pasivos que afectan al patrimonio neto, excluyendo operaciones con los propietarios. Esto supone un acercamiento al concepto de resultado económico, defendido por los autores de la escuela clásica normativo deductiva, aunque en la actualidad no concebido como una magnitud a priori única e incontestable, sino pensado para satisfacer las necesidades de los usuarios, en particular las de los inversionistas.

Sin embargo, de acuerdo con el enfoque del excedente sucio, el resultado contable es el indicador del rendimiento de la empresa y de quienes la administran, fundamentado en las transacciones que lleva a cabo la misma y en el principio de realización bajo el modelo del costo histórico, lo que a su vez entronca con los planteamientos propugnados por los autores adscritos a la escuela clásica positivo inductiva.

En el marco de esta antigua controversia — desde la década de los noventa del siglo XX con la irrupción del valor razonable para la valoración de determinados 
instrumentos financieros y derivados, junto con la aprobación de los normas que regulan el resultado global por los principales organismos emisores de normas contables en el nivel internacional y particularmente en la actualidad con la revisión de la NIC 1 del IASB en el año 2007- asistimos a un claro posicionamiento a favor del enfoque del excedente limpio.

Esto supone que el resultado empresarial capture los cambios en el patrimonio neto no relacionados con operaciones con la propiedad, para con ello reportar una mayor relevancia a los inversionistas, considerados en el marco conceptual como usuarios de referencia. Para su revelación, según dicha norma, las empresas podrán optar por formular el estado de resultados tradicional de forma separada, e inmediatamente después el estado de resultados globales, que lo integra, o presentar únicamente este último estado contable. En este último caso estamos ante la desaparición del estado de resultados tradicional del conjunto completo de estados financieros periódicos de propósito general, y con ello ante el declive de los planteamientos propios del enfoque del excedente sucio.

Los anteriores cambios, que implican una nueva forma no ya de presentar sino también de concebir el resultado empresarial, hemos de entenderlos en el contexto actual de los modelos contables de corte anglosajón con una marcada orientación a la protección del mercado a los que tendemos.

Estas tendencias representan en la comunidad contable internacional un hecho de impacto significativo, en particular, para aquellos países como los latinoamericanos o los de gran parte de la Europa continental en los que sus modelos contables se han venido orientando esencialmente a la protección patrimonial y a elaborar una información con propósitos fiscales, con lo que entre otros elementos el estado de resultados tradicional estaba concebido para atender esas finalidades.

\section{Referencias}

Accounting Standards Board (ASB) (1992). Frs 3, Reporting Financial Performance, revisado en 1993, 1999 y 2007, ASB, London.

Alexander, S. S. (1950). "Income Measurement in a Dynamic Economy", Five Monographs on Business Income, Study Group on Business Income, New York: American Institute of Accountants (AIA). 
American Accounting Association, aAa (1936). "A Tentative Statement of Accounting Principles Affecting Corporate Reports", Executive Committee of the American Accounting Association, The Accounting Review, June, 187-191.

(1977). Statement on Accounting Theory and Theory Acceptance (soATATA), AAA, Sarasota.

American Institute of Accountants (aia) (1947). Accounting Research Bulletin, No. 32.

Association for InVestment Management And Research (AimR) (1993).

Financial Reporting in the 1990s and Beyond, a position paper prepared for Peter H. Knutson, AIMR (actual Chartered Financial Analyst Institute -CFA Institute-), Charlottesville.

Beale, B. y H. Davey (2000). "The Nature and Origins of Comprehensive Income”, en S. B. Dahiya (ed.), The Current State of Business Disciplines, Spell Bound Publications Ltd.,Vol. 1, 81-100, New Delhi.

Belkaoui, A. R. (2004). Accounting Theory, fifth edition, London: Thomson.

Brief, R. P. y K. V. Peasnell (1996). Clean Surplus: A link Between Accounting and Finance, Garland Publishing, Inc., New York and London.

Cañibano Calvo, L. y J. A. Gonzalo Angulo (1997). "Los programas de investigación en contabilidad", en Cañibano, Teoría actual de la contabilidad (Técnicas analíticas y problemas metodológicos), Ministerio de Economía y Hacienda, Madrid: Instituto de Contabilidad y Auditoria de Cuentas.

Cea García, J. L. (1994). El resultado contable. Análisis crítico de la medición del excedente económico empresarial, Madrid: AECA.

(2005). El marco conceptual del modelo contable IASB: Una visión crítica ante la reforma de la contabilidad española, Madrid: Centro de Estudios Financieros. 
Chambers, R. J. (1965). "The Development in Accounting Theory", en R. J. Chambers, L. Goldberg, y R.L. Mathews (Eds.), The Accounting Frontier, Cheshire, 18-35.

Chatfield, M. (1977). A History of Accounting Thought, New York: Krieger.

Dickinson, A. L. (1908). "IV Forms of Accounts", en "Accounting Practice and Procedures", The American Association of Public Accountants Twenty-First Anniversary Year Book.

EDWARds, E. O. y P. W. Bell (1961). The Theory and Measurement of Business Income, University of California Press, Seven Printing, 1973, Berkeley, Los Ángeles, London.

European Financial Reporting Advisory Group-Instituto de ContabiliDAd y Auditoría de Cuentas (EFRAG-ICAC) (2006). "The Performance Reporting Debate", PAAinE, Discussion Paper, disponible en http://www. efrag.org en el enlace Projects, PAAinE Projects, Performance Reporting, traducción al español disponible en http://www.aeca.es/performance_ reporting.pdf [accesos el día 4 de junio de 2008].

Federeral Reserve Board (1917). "Uniform Accounts”, Federal Reserve Bulletin, April 1, 284.

(1929). "Verification of Financial Statements" (revised), A revision by the American Institute of Accountants, of suggestions for the preparation of balance-sheet statements printed in the April, 1917, issue of the Federal Reserve Bulletin, United States Government Printing Office, 26.

Feltham G. A. y J. A. Ohlson (1995). "Valuation and Clean Surplus Accounting for Operating and Financial Activities", Contemporary Accounting Research, Vol. 12, Spring, 689-731.

Financial Accounting Standards Board (Fasb) (1975). SFas 8, Accounting for the Translation of Foreign Currency Transactions and Foreign Financial Statements", (reemplazado por el SFAS 52), disponible en http://www. fasb.org/st/ [acceso el día 4 de agosto de 2008]. 
(1981). SFAS 52, Foreign Currency Translation, FASB, disponible en http://www.fasb.org/st/ [acceso el día 24 de mayo de 2009].

(1985). SFAS 87, Employers' Accounting for Pensions, FASB, (rectificado por el SFAs 132), disponible en http://www.fasb.org/st/ [acceso el día 24 de mayo de 2009].

(1993). SFAs 115, Accounting for Certain Investments in Debt and Equity Securities, FASB, disponible en http://www.fasb.org/st/ [acceso el día 24 de mayo de 2009].

(1997). SFAS 130, Reporting Comprehensive Income, disponible en http://www.fasb.org/st/ [acceso el día 24 de mayo de 2009].

(2000). Statements of Financial Accounting Concepts (FASB), 2000/2001 Edition, en John Wiley \& Sons, Inc., New York, Chichester, Brisbane, Toronto, Singapore.

(2006). SFAs 157, Fair Value Measurements, disponible en http://www.fasb.org/st/ [acceso el día 24 de mayo de 2009].

(2009). "Final FASB Staff Positions": FSP SFAS 107-1 y APB 28-1, FSP SFAS 115-2 y SFAS 124-2, y FSP SFAS 157-4 (FASB Issues Final Staff Positions to Improve Guidance and Disclosures on Fair Value Measurements and Impairments), [acceso el día 24 de mayo de 2009].

(2009). Financial Statement Presentation, proyecto desarrollado conjuntamente con el IASB, disponible en http://www.fasb.org [acceso el día 24 de mayo de 2009].

García-Ayuso Covarsí, M. y J. Monterrey Mayoral (1998). "El modelo de valoración edwards-bell-ohlson (EBO): aspectos teóricos y evidencia empírica", Revista Española de Financiación y Contabilidad, Vol. XXVII, $\mathrm{N}^{\circ} 96,751-785$.

Gilman, S. (1939). Accounting Concepts of Profit, New York: The Ronald Press Company. 
Gonzalo Angulo, J.A. (1996): "La propuesta para 'dar valor a los informes de las empresas'(MCRV): Posibilidades de implantación en España de una contabilidad a valores de mercado", en Ensayos sobre Contabilidad y Economía, en homenaje al Profesor Ángel Sáez Torrecilla, Tomo II, Contabilidad de Gestión y Economía Financiera, Madrid: ICAC, 571-589.

(2000). "Criterios de valoración y mantenimiento del capital", en Túa, El marco conceptual para la información financiera. Análisis y comentarios, Madrid: Asociación Española de Contabilidad y Administración de Empresas (AECA), 225-261.

Hatfield, H. R. (1927). Accounting. Its Principles and Problems, New York: Appleton-Century-Crofts, Inc.

Hendriksen, E. S. (1982). Accounting Theory, Richard D. Irwin, Inc., Fourth Edition, International Edition, Homewood.

IJIRI, Y. (1975). Theory of Accounting Measurement, American Accounting Association (AAA).

International Accounting Standards Board, iasb (1989). Framework for the Preparation and Presentation of Financial Statements, London: IASC.

(1997). IAS 1, Presentation of Financial Statements, IASC, London, reemplazada por la IAs 1 (2003).

(2003) IAs 1, Presentation of Financial Statements, en Improvements to International Accounting Standards, 2003, IASB, London, reemplazada por la IAS 1 (2007).

(2007). IAs 1, Presentation of Financial Statements, en International Financial Reporting Standards, en Bound Volume 2008, London, IFRss ®, IASB, IASC Foundation.

(2009). Financial Statement Presentation, proyecto desarrollado conjuntamente con el FASB, disponible en http://www.iasb.org [acceso el día 24 de mayo de 2009]. 
Jones, S. y M. Aiken (1994). "The Significance of the Profit and Loss Account in Nineteenth-Century Britain: A Reassessment”, Abacus, Vol. 30, No. 2, 196-230.

Kam, V. (1986). Accounting Theory, New York: John Wiley \& Sons.

Kiger, J. E. y J. R. Williams (1977): "An Emerging Concept of Income Presentation”, The Accounting Historians Journal, Fall, 63-78.

Linsmeier, T.J., J. Gribble, R. G. Jennings, M. H. Lang, S. H. Penman, K. R. Petroni, D. Shores, J. H. Smith y T. D. Warfield (1997). “An Issues Paper on Comprehensive Income”, American Accounting Association's Financial Accounting Standards Committee, Accounting Horizons, Vol. 11, No. 2, 120-126.

Littleton, A. C. (1940). "The Integration of Income and Surplus Statements", Journal of Accountancy, January, 30-40.

LukKa, K. (1990). "Ontology and Accounting: The Concept of Profit", Critical Perspectives on Accounting, Vol. 1, 239-261.

Macneal, K. (1939). Truth in Accounting, Philadelphia: University of Pennsylvania Press.

Martínez García, F. J. y F. Sousa Fernández (2004). "La conceptuación del beneficio como exponente de la diversidad contable internacional", $\mathrm{X}$ Premio AECA para Artículos sobre Contabilidad y Administración de Empresas y del Sector Público, Revista de AECA, nº 67, 3-7.

Mattessich, R. (2002). “Teoría del excedente limpio y su evolución: Revisión y perspectivas recientes", Energeia: Revista Internacional de Filosofía y Epistemología, Vol. 1, No. 2, Buenos Aires: Universidad de Ciencias Empresariales y Sociales, Spanish Translation , 49-79.

May, G. O. (1950). "Business Income”, The Accountant, September 30, 315-323.

Moonitz, M. (1961). The Basic Postulates of Accounting, Accounting Research Study No. 1, New York: American Institute of Certified Public Accountants (AICPA). 
Mora Enguidanos, A. (2004). "La normativa internacional sobre instrumentos financieros: un debate sin resolver", Accésit del X Premio AECA para Artículos sobre Contabilidad y Administración de Empresas y del Sector Público, Revista de AECA, $\mathrm{n}^{\circ}$ 67, 8-10.

Paton, W. A. (1922). Accounting Theory, New York: The Ronal Press.

(1934). "Shortcomings of Present-Day Financial Statements", Journal of Accountancy, February, 127-128.

Paton, W. A. y A. C. Littleton (1940). An Introduction to Corporate Accounting Standards, Chicago: American Accounting Association.

y R.A. Stevenson (1916). Principles of Accounting, The Ann Arbor Press, Ann Arbor, [Reprinted 1976, Arno Press, New York].

Sanders, T. H., H. R. Hatfield y U. Moore (1938). A Statement of Accounting Principles, American Institute of Accountants, AIA, New York, reprinted by the American Accounting Association (1977).

Sousa Fernández, F. (2007). El resultado global en el ámbito de la información financiera internacional: marco conceptual, análisis comparado de normas y un estudio empírico para grupos europeos cotizados en NYSE y NASDAQ [en línea], Tesis doctoral, Departamento de Administración de Empresas, Universidad de Cantabria, http://www.tesisenred.net/TDR-0430108-085640 [acceso el día 7 de agosto de 2008].

Sprouse, R. T. y M. Moonitz (1962). A Tentative Set of Broad Accounting Principles for Business Enterprises, Accounting Research Study No. 3, New York: American Institute of Certified Public Accountants (AICPA).

Storey, R. K. y S. Storey (1998). The Framework of Financial Accounting Concepts and Standards, Special Report, Financial Accounting Series, FASB, Norwalk.

Tribunal Supremo de los Estados Unidos (1920). "Sentencia del caso Eisner vs. Macomber", [25 USA188,195]. 
Túa Pereda, J. (1983). Principios y normas de contabilidad, Instituto de Planificación Contable, Madrid: Ministerio de Economía y Hacienda.

U. S. SECURITIES AND EXCHANGE COMMission (SEC) (2008). "Report and Recommendations Pursuant to Section 133 of the Emergency Economic Stabilization Act of 2008: Study on Mark-To-Market Accounting”, disponible en http://www.sec.gov/news/studies/2008/marktomarket123008.pdf [acceso el día 24 de mayo de 2009].

Walsh, E. (1995): "Current Status of Clean Surplus", Unpublished Paper, New York: University, 7.

Zeff, S. A. (1972): Foreign Accounting Principles in Five Countries: A History and Analysis of Trends, Stipes Publishing, Co., Champaign. 